\title{
BIM model-based design delivery: Tideway East, England, UK
}

Michael Gaunt MEng(Hons), CEng, MICE

BIM Manager, Mott MacDonald, London, UK

(michael.gaunt@mottmac.com) (Orcid:0000-0001-6057-3044)

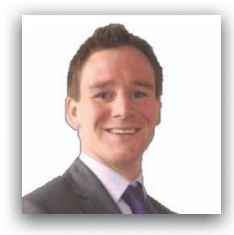

The UK Government Construction Strategy 2016-2020 aims to drive 'increased construction productivity to facilitate forecast efficiency savings of $\mathbf{f 1 . 7}$ billion over the course of this Parliament'. This strategy defines objectives which 'build on the success of the Government Construction Strategy 2011-2015' and the requirement for 'fully collaborative 3D BIM as a minimum by 2016'. PAS 1192-2:2013 supports this strategic objective and highlights the need for 'efficient and accurate information exchange' and along with BS 1192:2015 promotes the 'avoidance of wasteful activities'. The lean production of information is key to achieving this objective, ensuring that 'information can be used and re-used throughout the project' and reducing the 'over-production of information with no defined use'. Model-based delivery, in the context of this paper, describes a lean design delivery process which removes abortive drawing production. This process enables a reduction in programme and cost but requires all parties involved to develop improved model review skills to adopt this new method of working. This paper describes the implementation, benefits and challenges of model-based delivery on the eastern section of the Thames Tideway Tunnel.

\section{The challenge}

To ensure design development is controlled and managed, it is generally accepted that design data are submitted to project stakeholders for review at several key milestones before construction. These milestones are generally referred to as design gates or data drops and capture key development points on the design programme. As a result of multigated assurance processes, drawings must be reproduced from the design model at each gate for review purposes. It is this drawing production process which is considered abortive. Following gate submission, these drawings quickly become superseded as the design model continues to develop. The challenge, to improve design efficiency, is to remove these abortive tasks and focus all project stakeholders on the single source of design truth, the design model.

Model-based delivery, in the context of this paper, refers to a lean design delivery process in which abortive drawing production is eliminated and the design model becomes the true single source of information. Removing abortive drawing production during the design phase of a project has the potential to reduce programme and cost while improving collaboration and model engagement. This requires a developed level of technology skills across all project stakeholders, a reliable software platform and behavioural changes across all teams. This paper describes the implementation of model-based delivery on the Thames Tideway Tunnel eastern section, the benefits realised and the challenges encountered.

\section{The Thames Tideway Tunnel project}

The Thames Tideway Tunnel is a major infrastructure project in London; it is designed to reduce the flow of untreated sewage to the River Thames. It consists of a large interception and conveyance tunnel which is connected to the existing sewer network at 24 interception sites. A diagram of the project is shown in Figure 1.

Mott MacDonald is the lead designer for the CVB JV (a joint venture of Costain, Vinci Construction Grands Projets and Bachy Soletanche) responsible for the east contract from Chambers Wharf to the Abbey Mills pumping station. This contract also includes the Greenwich Connection Tunnel running from Greenwich to Chambers Wharf.

The Thames Tideway Tunnel project has an aspiration to be transformational in every sense. This approach was evident in the initial 6-month optimised contractor involvement (OCI) process which allowed challenges to the work information (WI) to deliver efficiencies on the project. One of the main challenges was to reduce the thousands of required deliverables into fewer, coordinated packages of information. Model-based delivery was the response to this challenge, and open-mindedness and willing approach from all parties allowed this method to be implemented.

The delivery methodology implemented on the Thames Tideway Tunnel consists of six design gates; this is shown in Figure 2. 


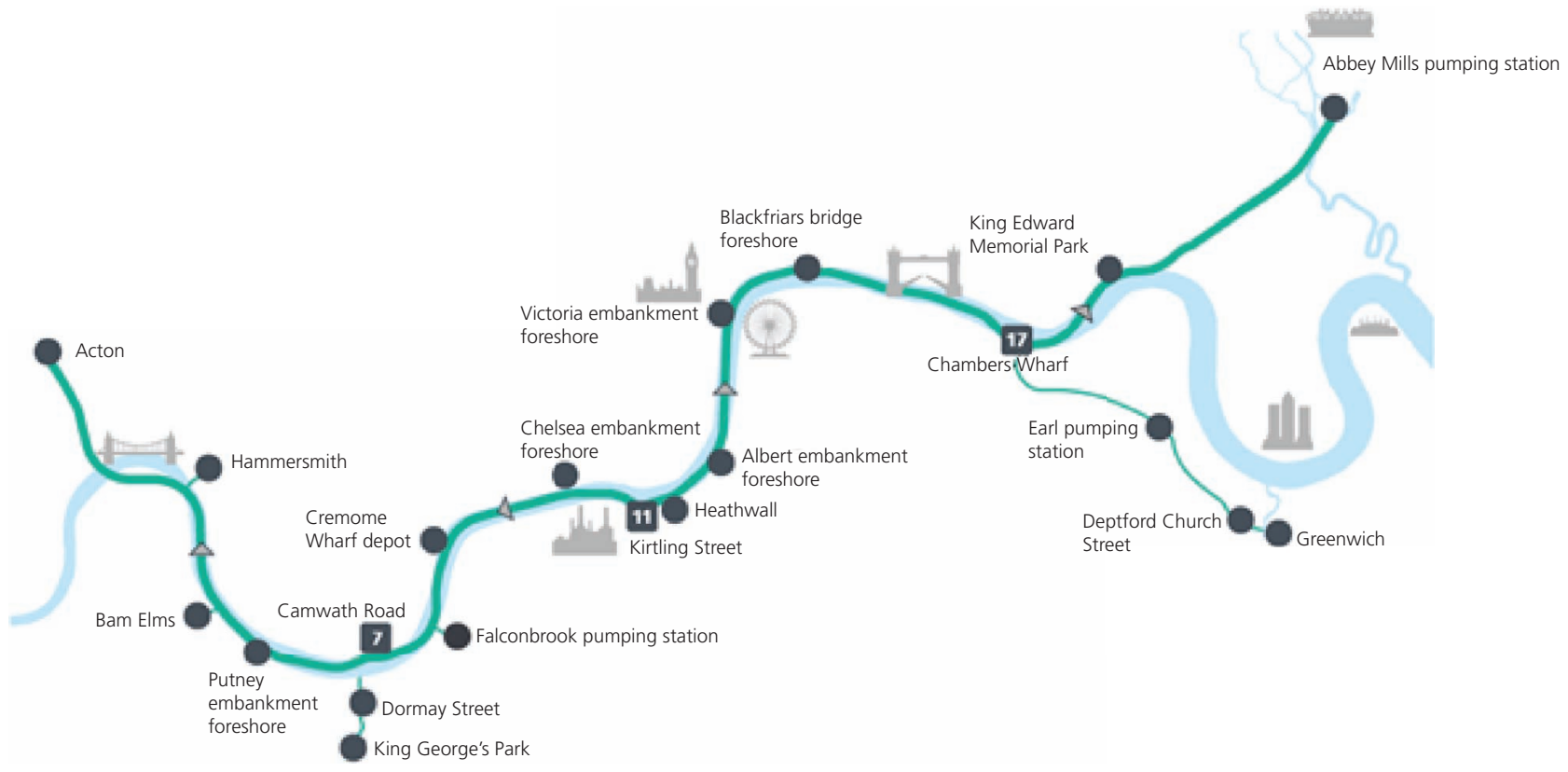

Figure 1. Thames Tideway Tunnel main work sites

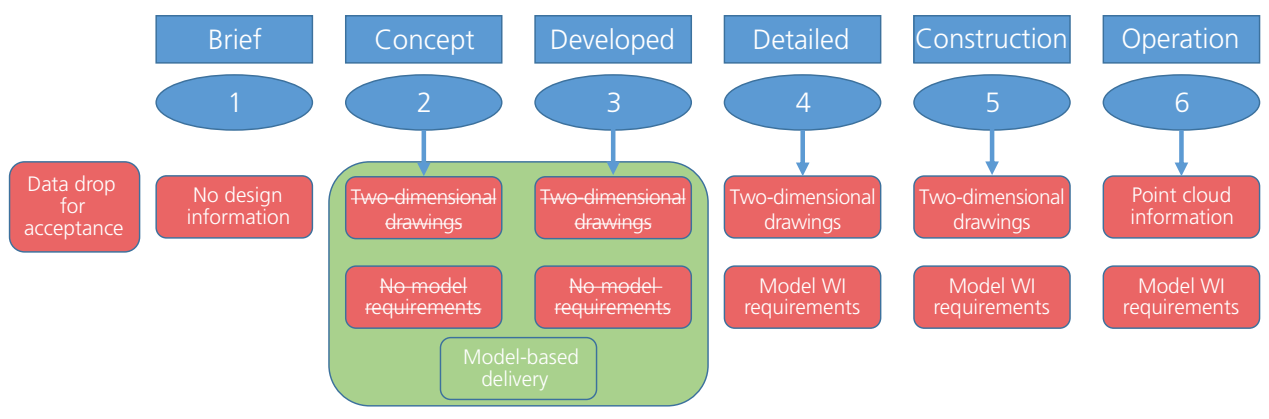

Figure 2. Gated assurance process. Items shown in red are WI requirements; items shown in green were implemented as part of the model-based delivery concept. PDF, portable document format

Each design gate requires an increased level of detail before construction is approved at gate 5. Model-based delivery was established as the agreed design delivery procedure for gates 2 and 3 only.

\section{Drawing-based delivery}

To review the benefits of model-based delivery, comparison will be made to the currently generally accepted drawing-based delivery method. This method consists of the development of federated design models in a three-dimensional (3D) environment followed by the generation of two-dimensional (2D) drawings; this is shown in Figure 3.

In compliance with BS 1192:2015 (BSI, 2015), 3D models are regularly shared until a coordinated design model has been produced. At this point, the design development is placed on hold, typically 2 weeks before design submission. The focus of the design team then switches to drawing production. Two-dimensional drawings are created which sufficiently demonstrate the design intent with dimension and annotations compliant with project computer-aided design (Cad) standards. Each 3D and 2D model is then subjected to an engineering and coordination check, before delivery of the final package of information. Any comments requiring a design change because of the design review process would require this entire process to be revisited from the start.

This delivery mechanism, combined with a gated assurance process and an acceptance and commenting process, shrinks the productive design period to a small part of the process. As shown in Figure 4, relying on the creation of drawings for review not only lengthens the design process but also delays the intervention from other parties until the production of drawings is complete. 


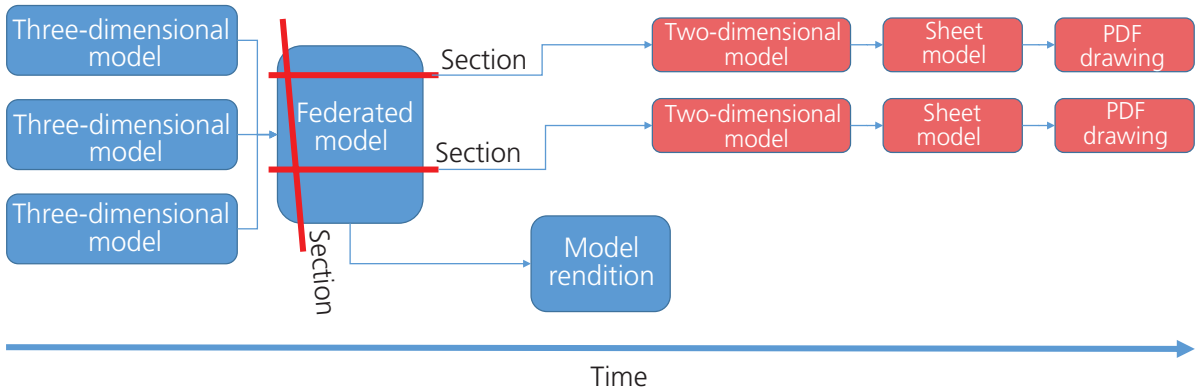

Figure 3. Traditional drawing delivery process (drawing production shown in red)

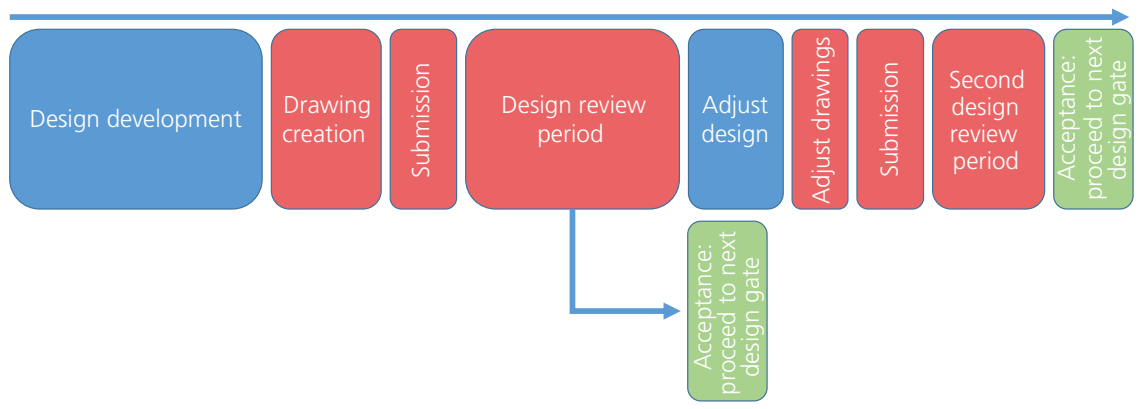

Figure 4. Traditional delivery mechanism. Productive design development is shown in blue

Constraints on programme and cost are regularly encountered in this process; it is not uncommon for a designer to be left in a position whereby the $3 \mathrm{D}$ models must continue to develop while the $2 \mathrm{D}$ drawings are under review. The many stakeholders of the project team are then working on a variety of information sources - this is not the single source of truth.

\section{Model-based delivery}

Model-based delivery aims to enhance the use of technology to reduce non-productive design periods. Model-based delivery drives efficiency by ensuring the model is the single source of design truth; no longer can the model be left in the domain of Cad technicians. While this requires additional training and skill development, the perceived benefits that the project team expected were

reduced design delivery programme and resource requirements

- reduced ambiguity in information transfer

- improved design model engagement - the primary source of information

- enhanced future opportunities for efficient information transfer - for example, four-dimensional (4D) modelling and digital markups.

Perhaps, the greatest benefit, however, is the reduction in the design change workflow when changes are made directly to the $3 \mathrm{D}$ model rather than creating $2 \mathrm{D}$ drawings. Review is an iterative process; until the final product is agreed, the length of this workflow must be minimised where possible. Figure 5 shows the significant reduction in the design change process that modelbased working can bring.

Model-based delivery combined with collaborative working methods allows all involved in the design process to monitor the evolution of the design, gaining confidence as the design progresses. Removing the $2 \mathrm{D}$ drawing production allows the 3D model to be 'dropped' to the client as soon as the design is complete; the review processes can be reduced further if the client is fully engaged in the development process. For example, collaborative design review sessions were held on a weekly basis allowing all project stakeholders to engage with the design. In addition, Tideway used software to review the design models and produce digital markup files. These files signposted the exact location of the issue in the 3D model reducing the likelihood of miscommunication in the commenting process. Model-based delivery facilitates collaborative working in contrast to a series workflow and infrequent information transfer which 2D drawing production demands. While examples exist of projects within construction which have adopted the model as the primary source of information transfer (steelwork fabricators, Design for Manufacture and Assembly plants etc.), building information modelling (BIM)-only delivery is not common on major construction projects due to the risks, unknowns and lack of skill sets associated with this new process. Recent 


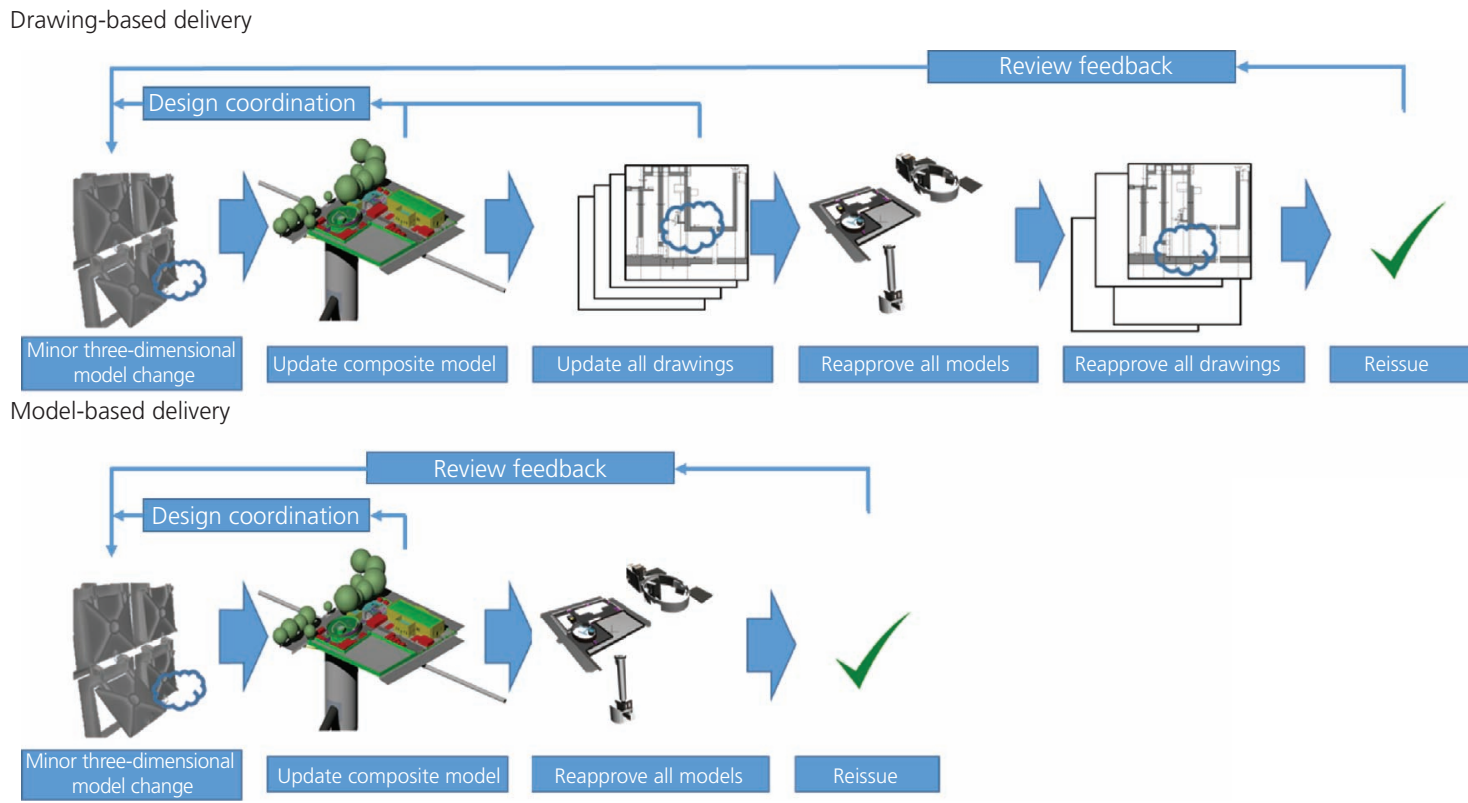

Figure 5. Significantly reduced design change process when adopting 3D models as the focus of design reviews

contractual requirements such as the Construction Industry Council BIM protocol will drive the need for this delivery method: 'in the case of conflicting information, the BIM model shall prevail'.

\section{Literature review}

As part of this paper, the author has completed a literature review of previous best practices in this area. The literature review focuses on the theory behind the paper, that creating $2 \mathrm{D}$ information for communication purposes can be removed to improve design efficiency. While current projects may now be adopting similar approaches, the use of drawingless or model-only delivery is scarce in recent BIM and civil engineering literature. Similarly, while project savings from the use of 3D models for communication during the construction and operation stages are widely available and hypothesised, savings during the design production are not well documented. The complex impacts of design change and potential contractual restrictions in a commercial environment may, in part, be the reason for this. The Smisto project (a hydropower scheme in Norway) is a rare example of publicised drawingless design within civil engineering. While no quantifiable feedback is provided, "[t]he advances of the Smisto project has convinced the main contractor Hæhre Entreprenør AS and Multiconsult that drawingless execution is worth pursuing' (Multiconsult, 2016). In the UK, Crossrail has driven the use and upskill of BIM processes across the industry; however, "no consideration was given to 3D models being a formal contract deliverable (in 2007)' (Smith, 2014: p. 146). This demonstrates the novelty of the Tideway East approach on a major infrastructure project and the need to share this approach with the rest of the industry.
The perceived benefits of BIM-based working are regularly highlighted in industry literature, advertising material and social media. 'Reports forecast that the wider adoption of BIM will unlock $15-25 \%$ savings to the global infrastructure market by 2025' (EU BIM Task Group, 2017: p. 4). The UK BIM Task Group reports that 'based on the UK Government's construction benchmarking report, its BIM programme contributed to combined savings of $£ 2 \cdot 2 \mathrm{bn}(\$ 3 \cdot 1 \mathrm{bn})$ between 2013 and 2015, making it a significant tool to meet its target of $15-20 \%$ savings on public construction costs' (BSI, 2017: p. 1). The perceived benefits of using a model-based approach on Tideway East were estimated to be in the region of $20 \%$ based on a comparison between the lengths of the existing and the proposed workflows. This compares favourably to the industry reports previously discussed.

The level of BIM maturity is the 'ability of the construction supply chain to operate and exchange information' according to the BS 1192-4:2014 standard (BSI, 2014: p. 1). 'This standard defines expectations for the exchange of information throughout the lifecycle of a Facility' (BSI, 2014: p. 1); however, it does not define the expectations of information transfer for design assurance. Meanwhile, 'PAS 1192-2 focuses specifically on project delivery' (BSI, 2013: p. 1) and states that '[p]ost contract award, the purpose of the BIM Execution Plan is to facilitate the management of delivery on the project. This includes the contractual information exchange requirements set out in a BIM protocol alongside the wider project deliverables established by the contract' (BSI, 2013: p. 16). It does not make a specific reference to the information format during the design assurance phase. 'One of the key Level 2 requirements is the exchange standard of COBIe and PDF, as well as copies of the native files' (BSI, 2013: p. viii). Tideway East has 
exceeded this requirement by removing 2D PDF drawing files and replacing this deliverable with the native, single-source-of-truth model file. The project has adopted the BS 1192:2015 approach of 'the avoidance of wasteful activities such as ... over-production of information with no defined use' (BSI, 2013: p. iv).

The model-only design delivery is embedded within the aerospace and automotive industries. For example, the model-based enterprise (MBE) engineering strategy has been around for some time in the USA and the Department of Defense has been working to implement this throughout its supply chain. 'Studies conducted by the U.S. Department of Defense and various commercial suppliers estimate that up to one-third of all money spent by engineering budgets is allocated to the creation and maintenance of $2 \mathrm{D}$ drawings during the initial production cycle and to support long-term product sustainment' (Solidworks, 2015: p. 3). The advantages of MBE as set out in the MBE Summit Report (National Institute of Standards and Technology, 2013) are as applicable to civil engineering as to aerospace and manufacturing. BIM processes are based on 'beginning with the end in mind ... and identifying the downstream uses of information, to ensure information can be used and re-used throughout the project and life of the asset' (BSI, 2013: p. iv). Model-based delivery encapsulates this strategy, and Tideway East serves as a unique demonstration of this approach on a major project.

\section{Project set-up and planning}

To implement the model-based delivery concept, all parties worked closely together to assess the advantages and risks to the project. This assessment was captured as an alliance-level initiative during the initial OCI stage, ensuring all main work contractors, Tideway and Thames Water, believed in the benefits and the methodology was crucial to the success of the initiative.
While the Thames Tideway Tunnel project desired to be transformational, the impact of failure could be significant on the project and the industry. The risks include (due to limited experience and lesson sharing across the industry) but are not limited to those shown in Table 1 .

The major risk was assessed to be the acceptance of a noncompliant design due to a failure to change the project culture and upskill all associated parties to the necessary levels, resulting in a failure to review the model information. To mitigate this risk and bring together project teams in a more collaborative manner, weekly review sessions were set up on Wednesdays. These sessions provided a forum for all project participants to discuss key project issues and agree on solutions. Bentley Navigator was the preferred review tool to interrogate the latest shared model information and capture comments.

The implementation of model-based delivery required a structured review of project delivery processes. Linking the design management plan, BIM execution plan (BEP) and information management plan was crucial in ensuring that the delivery technology was mandated throughout the project, to facilitate digital checking, approving, federation and reviews. This is shown in Figure 6.

A month of software and process testing was undertaken prior to the start of the project, and a structured training programme was also implemented within the design team. The testing immediately identified that the lack of embellishments and notes on a model in contrast to that on a drawing made the model review process time consuming. Only experienced model reviewers had the capability to measure difficult geometry and verify design compliance within the design review software. A solution was developed: the

Table 1. Key risks associated with model-based delivery

\begin{tabular}{|c|c|c|c|}
\hline Risk description & Impact & Mitigation strategy & Probability \\
\hline $\begin{array}{l}\text { Failure to change the project culture and } \\
\text { upskill all project parties to enable } \\
\text { model-based design reviews }\end{array}$ & $\begin{array}{l}\text { Acceptance of a non-compliant designs } \\
\text { resulting in changes and potential } \\
\text { rework later in the programme, } \\
\text { increased programme and costs. }\end{array}$ & $\begin{array}{l}\text { Weekly model-based design reviews to } \\
\text { engage all project parties and discuss } \\
\text { design issues and agree on solutions }\end{array}$ & Possible \\
\hline $\begin{array}{l}\text { Reduced design engagement from design } \\
\text { disciplines and project stakeholders due } \\
\text { to inefficient training, experience or } \\
\text { provision of hardware }\end{array}$ & $\begin{array}{l}\text { Design non-acceptance or non- } \\
\text { compliance leading to rework and } \\
\text { increased programme }\end{array}$ & $\begin{array}{l}\text { Assessment of existing skill levels and } \\
\text { hardware provisions } \\
\text { Implementation of extensive training } \\
\text { programme, and embed experts within } \\
\text { stakeholders to drive training }\end{array}$ & Possible \\
\hline $\begin{array}{l}\text { Loss of design data, file corruption or } \\
\text { uncoordinated designs because of } \\
\text { interoperability issues and the use of } \\
\text { untested software }\end{array}$ & $\begin{array}{l}\text { Increase in programme and design } \\
\text { rework as a result of lost data } \\
\text { Potential to revert back to traditional } \\
\text { delivery methods during the production } \\
\text { period with impact on programme and } \\
\text { cost }\end{array}$ & $\begin{array}{l}\text { Implementation of software testing plan } \\
\text { and common data environment (CDE) } \\
\text { system across all design parties to ensure } \\
\text { information sharing in compliance with } \\
\text { BS 1192:2015 }\end{array}$ & Unlikely \\
\hline $\begin{array}{l}\text { Legacy issue with future software formats } \\
\text { and versions including technology } \\
\text { failure (software or hardware). } \\
\text { Design that cannot be accessed due to } \\
\text { future compatibility issues is produced. }\end{array}$ & $\begin{array}{l}\text { Increased costs and programme impact } \\
\text { as design is reworked or converted to } \\
\text { other file formats }\end{array}$ & $\begin{array}{l}\text { Engagement with software vendors to } \\
\text { ensure continued compatibility in the } \\
\text { future }\end{array}$ & Unlikely \\
\hline
\end{tabular}




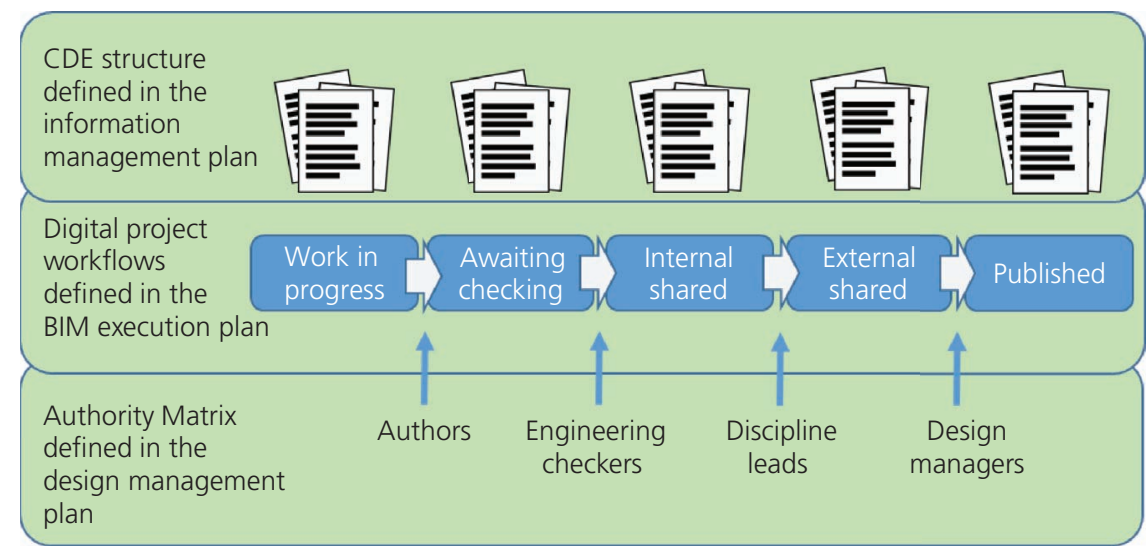

Figure 6. Integrating digital delivery processes throughout the project was crucial to control and manage the model information

delivered models would contain embedded annotations and notes to aid the review process. An example is shown in Figure 7; Bentley Dynamic Views were used to bring 2D notes into the 3D environment.

The Dynamic Views provided some of the dimensional data and notes that would traditionally be found on a $2 \mathrm{D}$ drawing, managing the culture change between drawings and models. Information previously found on a 2D drawing is therefore easily accessible and focused the engineering review on the model. Further dimensional data could be extracted using measuring tools in addition to any data requested to be embedded within the models. A model for each site was delivered at gate 2, following client review; comments were provided through a digital markup overlay file or an Excel spreadsheet.

While the process was considered a success at gate 2, software issues and the need to prepare for drawing delivery represented a production risk on the tight design programme. As a result, Dynamic Views were replaced by more traditional 2D extractions at gate 3 (see Figure 8).

A 2D model referenced back into the delivered federated model is shown in Figure 8. This method reduces the number of

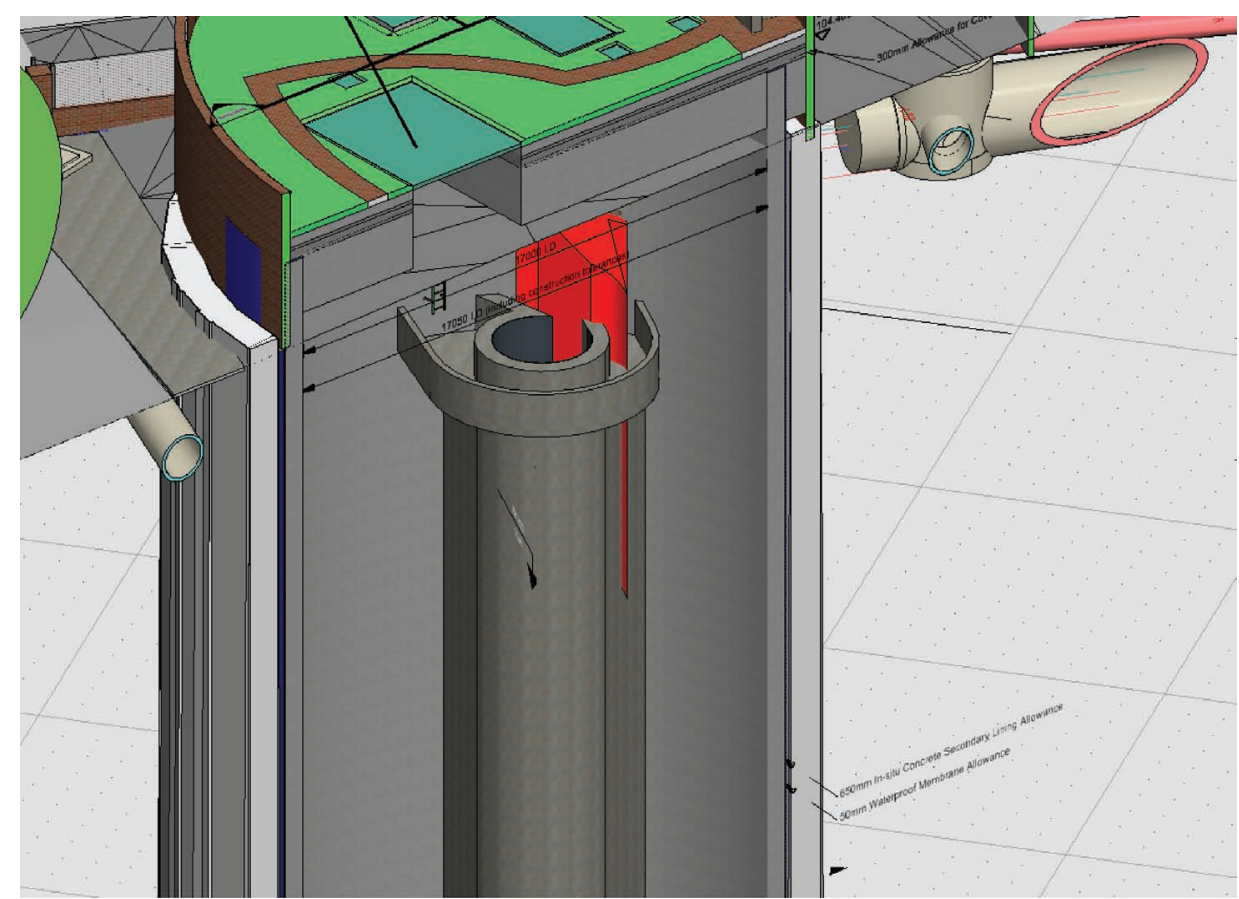

Figure 7. Model file with embedded Dynamic View annotations 
BIM model-based design delivery:

Tideway East, England, UK

Gaunt

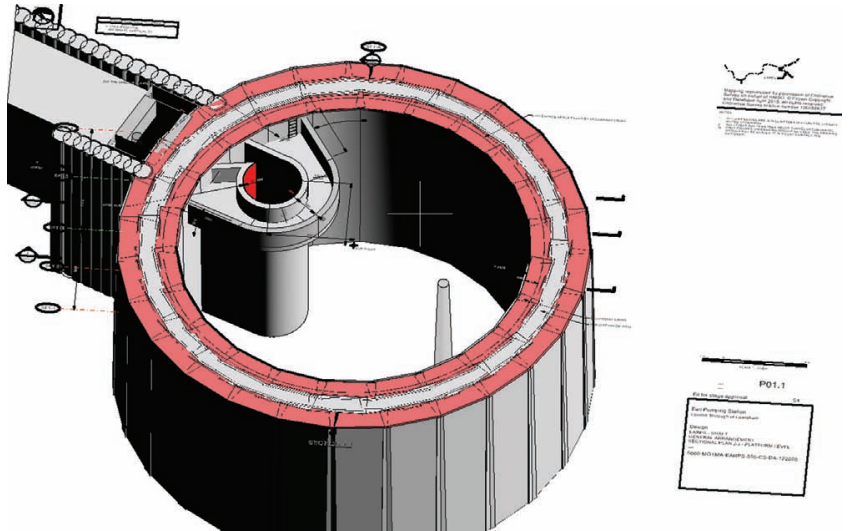

Figure 8. Federated model with an embedded extraction (note the drawing title block)

deliverables significantly, not only maintaining full access to the 3D model but also minimising the transition to more traditional 2D drawing production at gate 4 .

\section{Model-based delivery benefit analysis}

Following the delivery of design gates 2 and 3, information was extracted to review the impacts of the process and validate the predicted $20 \%$ workflow saving during the OCI period. Due to the bespoke nature of engineering projects and delivery mechanisms, obtaining a representable random control group was difficult. A comparison has therefore been produced between the actual production efforts for all modelling works and the perceived efforts (based on previous project data) of the more traditional drawing-based delivery.

Figure 9 shows the predicted savings between the federated model production efforts and the perceived efforts required to convey the

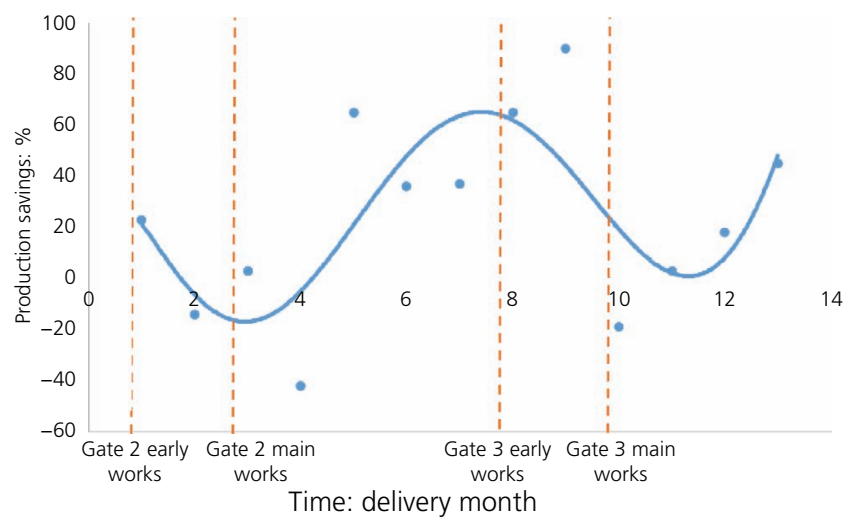

Figure 9. Savings in production efforts in comparison to drawing delivery for each deliverable at gates 2 and 3. Early works - diaphragm wall and base slab only. Main works - all design disciplines same engineering information using drawings. Each data entry represents a site model deliverable at design gate 2 or 3 for main works or early work design. Note that not all sites had a requirement to deliver an early work package of design; this was driven by the need to submit information early to meet the critical programme of the tunnel-boring machine. The number of dynamic views (gate 2) or extractions (gate 3 ) in the model has been used to quantify the number of drawings required for each delivery and, therefore, the perceived effort for drawing production. The information shown in Figure 9 is arranged based on the issue sequence - that is, the programme position. The results show a clear trend between programme position and production efforts; this demonstrates the learning curve associated with this method and the potential for greater future savings.

The gate 3 results shown in Figure 9 show higher average savings than the gate 2 results but with greater variation across the sites. This is driven by the variation in design complexity across the sites, accentuated at gate 3 as the design detail develops. Greater design complexity requires a higher number of extractions or drawings to convey the design details. This, therefore, leads to higher perceived savings as more drawings would be required in contrast to the single-model deliverable.

The results clearly show that model-based delivery results in lower design resource requirements because of the removal of abortive drawing production. On average, an efficiency savings of $32 \%$ can be demonstrated across the project at design gates 2 and 3. Further savings are likely to have been achieved through secondary factors such as reduced document control requirements and printing costs across all project parties.

While data have been collected to assess the potential resource requirements, programme impact is much harder to quantify as the project has been resourced to meet the design programme. While production information alone has been used to quantify efficiency improvements achieved because of this delivery method, it should be noted that model-based delivery supported the accelerated design and build delivery programme (2-year saving) on the Thames Tideway Tunnel project.

Table 2 gives a summary of the outcomes achieved compared to the perceived benefits at the start of the project.

\section{Feedback and challenges}

A questionnaire was distributed to the design and construction team following gate delivery. Obtaining feedback was crucial to assess issues with the process and evaluate the advantages to the team. The gate 2 questionnaires received a $42 \%$ response rate and delivered several key findings.

- The team generally responded positively to model-based submission and agreed that it should be adopted in future projects. The team strongly agreed that it significantly aided coordination. 
Table 2. Comparison between perceived project benefits and outcomes achieved

\begin{tabular}{|c|c|c|}
\hline Perceived benefits to the project & Outcomes achieved & \\
\hline $\begin{array}{l}\text { Reduced design delivery programme and } \\
\text { resource requirements }\end{array}$ & $\begin{array}{l}\text { Model-based delivery supported the accelerated ( } 2 \text {-year saving) delivery programme. Efficiency } \\
\text { savings of } 32 \% \text { were demonstrated at design gates } 2 \text { and } 3 \text { when compared to traditional } \\
\text { drawing delivery methods. }\end{array}$ & $\checkmark$ \\
\hline $\begin{array}{l}\text { Reduced ambiguity in information } \\
\text { transfer }\end{array}$ & $\begin{array}{l}\text { Model-based delivery combined with the CDE gave clarity to the design status. Model-based } \\
\text { design reviews allowed project teams to focus discussions on the models during the design } \\
\text { process rather than on the drawings at the end of the design gate. The design complexity at } \\
\text { gate } 3 \text { was communicated using one coordinated design model in contrast to many 2D } \\
\text { drawing deliverables. }\end{array}$ & $\checkmark$ \\
\hline Improved design model engagement & $\begin{array}{l}\text { Model-based delivery drove engagement on the design model; significant improvement in skill } \\
\text { levels and model-based checking; refer to team feedback from the questionnaires. }\end{array}$ & \\
\hline $\begin{array}{l}\text { Enhanced future opportunities for } \\
\text { efficient information transfer }\end{array}$ & $\begin{array}{l}\text { Models subsequently used for } 4 \mathrm{D} \text { model production, virtual reality operational scenarios and } \\
\text { temporary work coordination. It is inconclusive whether these opportunities can be directly } \\
\text { attributed to model-based delivery; however, focusing the teams on the model is likely to have } \\
\text { increased awareness. }\end{array}$ & - \\
\hline Reduced design change workflow & $\begin{array}{l}\text { Reduced workflows were evident within the design teams as changes were made based on } \\
\text { model reviews. Refer to team feedback from the questionnaires. }\end{array}$ & $\checkmark$ \\
\hline
\end{tabular}

- Teams that had a high reliance on coordination with other disciplines generally responded more positively.

- Identifying issues in the model environment rather than in 2D drawings was acknowledged to reduce the change process.

- The skills to work in the 3D model environment varied across the project team. More training was requested, and the model review process was noted as difficult due to the lack of intuition in the software.

The initial feedback from the client on the gate 2 models was positive, but it was apparent that there were several challenges to overcome before the process could be considered a success. These challenges can be summarised as follows.

- Improve model interrogation skills across the project.

- Ensure all have access to the correct software and hardware with appropriate licences.

- Improve understanding of the principles of the approach.

- Improve understanding of the model level of development.

A further questionnaire was distributed following gate 3 submission. From a $57 \%$ response rate, it was evident that as the design team expanded and the model level of detail increased, far greater model engagement had been achieved. While the responses demonstrated positivity towards the entire concept and willingness for the concept to succeed, they also identified a need for future development in the following key areas: software, skills and process consistency.

With regard to software, the available market software is not considered to be sufficiently intuitive to allow engineers to review detail quickly. The software requires a significant skill level to delve into the model with confidence and quickly extract details. User interfaces generally offer many more options than the standard engineer requires. The interface should align to the requirements of an engineer, not a Cad modeller.
With regard to skills, while the industry contains isolated pockets of excellence, the average skill level is not sufficient to implement digital processes effectively across a large project. Formalised training at all project levels should be mandated; however, this should be based on engineering principles. Software training could, in part, be assistance by the implementation of more intuitive software packages.

With regard to consistent processes, the recording of check information, markups and approval information should be consistent to all design disciplines and should be software independent. Currently, engineers must adopt the most appropriate software to the discipline due to the bespoke nature of discipline design. This, consequently, develops variety in checking methods because of the variations in authoring packages; further training is required as a result.

Respondents recognised the need for change and continued to believe that future industry projects should adopt this concept. The responses demonstrate that disciplines with a need to coordinate gained significantly from the additional focus on the model environment. This process improved design understanding and has driven the improvement of skills. Across the project, disciplines that would traditionally be far removed from the design process had the opportunity, from the start, to view the model. Importantly, they were forced to engage with the 3D models on the knowledge that $2 \mathrm{D}$ drawings would not be produced later.

\section{Recommendations}

For model-based delivery to be achieved in future projects, the following steps are recommended.

- The delivery method should be reviewed and adopted at the start of the project. Beyond this point, the opportunity to implement a culture change is significantly reduced. It should be supported through strong leadership and a clear vision. 
Smart Infrastructure and Construction

Volume 170 Issue SC3
BIM model-based design delivery:

Tideway East, England, UK

Gaunt
- The early availability of model-based information drives engagement and collaboration, but it should be supported by a thorough training plan across project parties, not just the authoring team.

- The method to extract information and review models should be defined at the start. Is embedded dimensional data within the model sufficient? Are 2D annotations really required?

- Software vendor engagement should be considered at the start of a project, and an assessment of the most appropriate reviewing tool should be undertaken by engineers.

- Include an opportunity and value section in the BEP which clearly describes positive steps to improve standard process. Ensure improvement is a requirement.

- Be flexible, for technology will encounter issues. Develop a culture which embraces yet manages the risk of new technology.

\section{Conclusion}

The implementation of model-based delivery on the Thames Tideway Tunnel project has demonstrated that greater efficiency in design delivery has been realised through the removal of abortive drawing production. This has driven far greater levels of engagement on the digital design models and subsequently reduced the design delivery and change process. Savings have been demonstrated at early design gates when compared to more traditional delivery methods, and further savings are predicted at subsequent design gates. Working in a model-only environment has also brought the project teams closer together to gain a greater understanding of the design earlier in the design process. Combined with a progressive assurance approach, it has improved collaboration across all parties.

While the concept has been demonstrated, further development is required to realise the true value of this process. Skill levels and engagement across all parties and the industry must improve, but this must be supported with software that meets the need of engineers who approve and review each design model. This software should be intuitive, format and system agnostic and auditable and should integrate fully with the CDE. It should be as easy to extract model dimension data as it is to use a shopping self-checkout machine.

Model-based delivery demonstrates the Tideway objective to be transformational in every sense. The implementation of this approach would not be possible without open-mindedness and collaborative and willing approach from all parties. This approach has allowed the use of technology to prevail and leaves a legacy of knowledge within the client, contractor and design teams. While ultimately, most construction work is based on primitive 2D drawing technology, demonstrating these savings during the design period gives significant cause for future optimism. The savings realised during design are insignificant in comparison to the potential for savings during construction. As more projects adopt model based-delivery, skills and technology will improve and model-based delivery will eventually become the status quo for site information. For now, it is up to the industry to embrace, engage and share knowledge to realise the benefits of modelbased delivery.

\section{REFERENCES}

BSI (2013) PAS 1192-2:2013: Specification for information management for the capital/delivery phase of construction projects using building information modelling. BSI, London, UK.

BSI (2014) BS 1192-4:2014: Collaborative production of information. Fulfilling employer's information exchange requirements using COBie. Code of practice. BSI, London, UK.

BSI (2015) BS 1192:2015: Building information management - a standard framework and guide. BSI, London, UK.

BSI (2017) BIM Level 2 Guidance, UK edn. BSI, London, UK. See http://bim-level2.org/globalassets/pdfs/international-guide-chapter-1introduction.pdf (accessed 29/11/2017).

EU BIM Task Group (2017) Handbook for the Introduction of Building Information Modelling by the European Public Sector. EU BIM Task Group, London, England, UK. See http://www.eubim.eu/wp-content/ uploads/2017/07/EUBIM_Handbook_Web_Optimized-1.pdf (accessed $10 / 12 / 2017)$.

Multiconsult (2016a) Building a Hydropower Station without Any Drawings. Multiconsult, Oslo, Norway. See http://www. multiconsultgroup.com/building-a-hydropower-station-without-anydrawings/ (accessed 23/07/2017).

National Institute of Standards and Technology (2013) Model-Based Enterprise Summit Report. National Institute of Standards and Technology, Gaithersburg, MD, USA, NIST Technical Note 1820. See http://nvlpubs.nist.gov/nistpubs/technicalnotes/NIST.TN.1820.pdf (accessed 23/07/2017)

Smith (2014) Building information modelling - moving Crossrail, UK, forward. Proceedings of the Institute of Civil Engineers Management, Procurement and Law 167(3): 141-151, https://doi.org/ 10.1680/mpal.13.00024.

Solidworks (2015a) Improve Quality, Reduce Costs, and Increase Efficiency with Model-based Definition. Solidworks, Waltham, MA, USA. See https://files.acrobat.com/a/preview/7053d00a-429f-472db337-65f5a78d2f18 (accessed 23/07/2017).

\section{How can you contribute?}

To discuss this paper, please email up to 500 words to the editor at journals@ice.org.uk. Your contribution will be forwarded to the author(s) for a reply and, if considered appropriate by the editorial board, it will be published as discussion in a future issue of the journal.

Proceedings journals rely entirely on contributions from the civil engineering profession (and allied disciplines). Information about how to submit your paper online is available at www.icevirtuallibrary.com/page/authors, where you will also find detailed author guidelines. 